\title{
Research on the Realization of 3D Animation Propagation Based on the Internet
}

\author{
Jing $\mathrm{Pu}^{1}$, Rui Hu${ }^{1}$, Chen $\mathrm{Shi}^{1}$, Yuwei Xiang ${ }^{1}$ \\ ${ }^{1}$ Sichuan Agricultural University, Dujiangyan, Sichuan, China
}

Keywords: 3D Animation Propagation, Internet.

\begin{abstract}
In recent years, the continuous innovation of new media technology has injected new vitality into the 3D animation industry. The speed and quality of its propagation directly affect the visual perception of animation. This paper focuses on the characteristics of Internet multimedia communication, compares various engine technologies, carries out theoretical analysis and operation verification, and looks for the best pattern of manifestation for the propagation of vector 3D animation.
\end{abstract}

\section{Background}

The Present Situation and Trend of 3D Animation. The Present Situation of 3D Animation. With the rapid development of Internet information technology and the continuous updating and upgrading of animation software, the 3D animation industry has emerged as a booming industry of global economic development. Its influence is not only reflected in economy and culture, but also in the public life. It has been widely used in various fields.

Nowadays, the 3D animation technology in China is successful, but it is not perfect. This is not only because it lacks profession and innovation, but also due to the ability of domestic software research and development. 3DS MAX, Maya and other production software are popular. Their comprehensive software functions can hinder the development of the industry to a certain extent while meeting the needs of the design. Fortunately, more and more people have noticed the development of the 3D animation industry and are willing to invest more time and money to create more realistic and emotional animations.

The Trend of 3D Animation. The 3D technology has entered a new stage. The further improvement of the audience's aesthetic level makes the 3D animation with low-quality unable to meet the needs of the audience's artistic appreciation. Therefore, it is imperative to make more high-quality, more delicate and more emotional 3D animation. The extremely authentic effect of 3D animation is the real factor to attract people to watch. To achieve the best effect, the most advanced software technology and a high level of professional ability are quite necessary. It shows the importance of independent research and development. In addition to the technological gap between China and the overseas 3D animation, there is also a lack of innovation. Since China has the extensive and profound culture, there are a lot of story resource and elements can be used in the animation design. Thus it is imperative to extract the native cultural elements into the traditional cultural characteristics, and create animations with rich local characteristics. In the future, the 3D animation industry in our country will change the former trend of blindly following and copy. The production of 3D animation will be derived from life but higher than the life, so as to achieve the final purpose with the most real effect and emotion and create the animation with local characteristics.

The Characteristics and Present Situation of Internet Multimedia Communication. The Characteristics of Internet Multimedia Communication. The Internet multimedia provides a platform for network information propagation. As a medium of propagation, Internet multimedia can propagate texts, images, sound and other information through different mobile terminals. There are several characteristics:

Diversity of information: The development of Internet promotes the communication and sharing of network information, making the information storage of Internet media extremely huge. The diversity of information transmission channels and forms has created the diversity of information. 
Information can be stored, searchable, and easily copied: Internet media promotes information through links. Huge web databases allow people to quickly find information, copy and store valid information through keyword searches.

Strong subjectivity and interactivity: In Internet propagation, the forms become more and more rich. The media can use Internet technology, no longer limited to books, magazines, etc. Users can receive messages or independently retrieve information, and experience by browsing and operating. They can have comments and feedback according to their real cognitive experience. They can have direct two-way communication to some extent.

Strong timeliness and permeability: Compared with traditional media, the advantage of Internet media lies in its high efficiency of transmission speed, wide scope of influence and accurate and timely information transmission. The upgrading of mobile devices and the improvement of people's living standards have made the public more connected with the Internet. The Internet media has gradually penetrated into all aspects of public life.

Information "fragmentation": The rapid pace of life has gradually "fragmented" public life, so the information received by the public is also more diffuse. The portability of the mobile terminal makes the Internet media occupy a large amount of the time of the public, so that the information can be transmitted in their daily time.

The Present Situation of Internet Multimedia Communication. The rapid penetration and development of Internet economy has attracted the close attention of many domestic media. In the past two years, with the development of information technology and the popularization of terminal equipments, more and more media have been using the Internet to achieve the aim of expanding customers and promoting the platform. The media has upgraded the service quality, increased user experience and expanded enterprise influence with the diversity of Internet platform display mode. As a new form of propagation, new media of network is gradually becoming mature, and the fragmented lifestyle has gradually expanded its influence. According to the data, the consumption of new media of network shows a growing trend, while the traditional media users continue to decrease. With the expansion of new media technology market, more and more people are attaching great importance to the new media of Internet and working to create a more ideal new media operation mode.

In recent years, the problem of cyber-violence has been emerging and the Internet users is of varying quality. The spread of bad speech has brought new challenges to the operation of the new media. This requires the new media workers to strengthen the platform management, and guide the public in an appropriate way to conduct the green healthy network communication.

\section{The Implementation Scheme of Propagation Based on HTML5}

Technical Principles. HTML5 is the latest version of HTML. It not only has a new syntactic feature, but also provides rich multimedia to meet the needs of other technical support and plug-ins. Several of the technologies it uses in web applications are:

Canvas: It's a rectangular area consisting of pixels. We can use the canvas elements to draw lines, squares, circles, and other shapes in the area. With Canvas technology, we can draw vector graphics more efficiently and flexibly to create richer and more interactive web pages. These pages can be viewed on any browsers and devices that support the HTML5 language.

Audio and Video: HTML5 provides local support for video and audio elements. Users can listen to songs and watch videos without downloading the plug-ins. They can also use tags to embed audios and videos or use attributes to define the multimedia experience. The control attribute can add videos and SRC attribute points within a certain pixel size to the files. However since modern browsers do not support the same video codec, we should have multiple source files in different forms as a backup.

Web storage: It consists of Session Storage and Local Storage. Local Storage is persistent Storage. It can specify key-value groups to use a web browser for local storage. In this way, Even if you leave the site, close the Tab Control of the browser, or exit the browser, the locally stored data will remain unchanged unless the user deletes them. Session Storage is period Storage. The data will also be cleared when the Tab Control of the browser or the window is closed. Unlike Cookies, it does not 
require a server response and information will not be transmitted to a remote Web server, which makes local storage much faster and more secure.

Inner hyperlink SVG (scalable vector graphics): SVG is a vector graphics format. Not only does it have good interactivity, but it's also very dynamic. Based on XML, the description of graphical language enables it to fully support DOM, which means that every dynamic element in the DOM can be used, or it can be scripted for each element.

The Effect Test. Using HTML5-Mugeda as the experimental object, we made an effect test of a small icon animation. The small icon was produced by key frame animation, mask animation, deformation animation, and path animation.

Mugeda was animation-controlled by referencing the named key frame, which avoided the inconvenience caused by the change of frame number in the animation editing. Before starting, the material was firstly imported into the material library. Next, the key frame was inserted into the material and named. Then, another frame was selected and inserted after it, and the key frame animation was inserted at the time line. After that, adjusting the material's position, animation and motion path line in the position of the first frame. Then, once again, adjusting the way of the material movement, naming the layer, and repeating the steps above. At last, creating the music layer(insert music material in the position of the second frame), and then previewing the effect. Finally the animation of a small icon was finished.

\section{The Implementation Scheme of 3D Animations Based on Web3D}

Mainstream Web3d Technology. CULT3D: Cult3D is a new 3D network technology. It consists of three parts: the output plug-in, the designer and the browser plug-in. The Designer is the core part to make 3D images which has excellent image quality, running speed and good interactive performance. Through Cult3D, developers can build models based on sound, links, animations, clickable JAVA code, and can control their movements. In addition, it has a low requirement for hardware, so even a poorly configured device can be browsed smoothly.

VRML technology: VRML technology is applied in many fields nowadays. It forms a different virtual world through the open modeling language, and it uses the set of nodes to complete the transformation of the Internet from 2D to3D, giving people unprecedented 3D experience.

VIEWPOINT: Its advantage is that the files can be minimized. In the process of 3D data downloading, the transformation from low-precision rough model to high-precision complex model can be completed. This proves its good transmission characteristics. It is composed of many medium elements such as 3D objects, textures, animations, interactive actions, and the definition texts of scenes. It has a high quality rendering engine that makes the effect real and does not require any hardware acceleration. Although the Viewpoint also has the ability of 3D modeling, it does not apply to building a simple model.

\section{The Implementation Scheme of 3D Animations Based on Flash}

Technical Principles. Flash finishes the production of animation by inserting the original provided by the component library through the key frame and add the relevant animation effects to the key frame and the time line. As a 2D animation software, FLASH has experienced a huge leap from making 3D model materials produced by the software which cannot directly support 3D animation to flash animation with 3D effects. The implementation ways of 3D animation made by FLASH mainly includes:

Using ActionScript to achieve 3D effects. It calculates the 3D coordinates, and then imitate them. The basic technical principle is to change the 3D coordinates into 2D coordinates and then calculate the epitope FLASH coordinates to achieve a 3D effect.

The rotation of the object elements in 3D space. This method is to import the elements in the element library to the stage. The animation command is then given to create a complete process of spinning motion in 3D space, which will complete the 3D effect of FLASH animation. 
FLASH animation is the use of the third-party software like 3Ds Max, Swift 3D, Maya, Dimension. Then importing the material they made with 3D effect into the FLASH in order to make the FLASH animation with 3D effect.

Through frame animation of FLASH. The frame animation of FLASH means producing the effect of 3D animation by changing the animation effect frame by frame. The effect of frame animation is simple. It has the basic principle which is close to the traditional animation, but it is more convenient than traditional production.

The Effect Test. The methods that set the role of the element library:

a. Create movie clip elements. Creating joint movie clip elements, and making clear of the relationship between the elements and joints. Adjusting the joints with any deformation tools until the joints are properly set.

b. The relationship setting of multistage joints. The bone chain is drawn from the root bone according to the position of the joint.

c. The linkage relationship setting between joints. The movement of sub-level joints in the default case will cause the movement of the advanced joints. But turning off the enabled option in the "Connect: Rotate" option of the attribute panel can fix the advanced skeletons.

$\mathrm{d}$. The setting of joint animation. For the joint animation setting, we can select "Insert" command. Then setting the key frame to the position of the corresponding frame serial number guided by the cursor. After that, Finishing the joint animation by selecting the use of the tool.

\section{Summary and Prospect}

The Summary. In the era of the rapid development of the Internet, 3D animation will become more and more widespread, and the data of 3D animation will become more and more. However, because of the 3D technology used in 2D animation, the data occupancy rate of the animation has greatly reduced. Meanwhile the production efficiency of 3D animation has been improved effectively. The production time and cost can also be reduced. At the same time, the propagation influence and quality of 3D animation on the Internet will improved.

The Prospect. In the development of the network 3D animation, because it is convenient to watch, and the time is short, the developing network $3 \mathrm{D}$ animation has a huge domestic market. At the same time, with the rapid development of the Internet and the age of big data, network animation is also developing rapidly, and its growing development is also the development trend of 3D animation.

\section{Acknowledgement}

Fund project: Subsidized by the social science key research base in Sichuan Province: the Research Center for Aesthetics and Aesthetic Education (15015).

\section{References}

[1]CSS3 extensions for setting web content in a 3D view volume and its stereoscopic 3D display[J]. Soon-Bum Lim,Hee-Jin Lee,Wen-Yan Jin,Seung-Min Shim. Computer Standards \& Interfaces.

[2] Research on Interactive Virtual Presentation Technology Based on WEB and CULT3D[A]. Digital Techniques • Humanized Design • Regional Autonomous Innovation_CAID\&CD'2006[C]. 2006.

[3] Yan-dong Rong, The Application in Web Page of 3D Technology Based on WebGL [J]. Information Security and Technology. 2015.08.

[4] Qing Pu. Realization of Interactive Network 3D Animation by JAS Development Technology Based on Cult3D [J]. Journal of Engineering Graphics. 2005.3.

[5] Dong Wang, Research on 3D Modeling and Animation Technology of VRML Based on Web3D[D]. Sichuan University, 2005. 